\title{
The Energy-Mass relation indicates that in remote times there was a great explosion
}

\author{
Enrique Martinez Viladesau \\ Escuela Técnica Superior de Ingenieros Industriales de Barcelona (ETSIUB), Spain \\ E-mail addresses: emviladesau@gmail.com
}

Received July 2019

Received in revised: Sept 2019

Published: Sept 2019

\begin{abstract}
When we read about an author who pretends to deduce the expression $E=m c^{2}$ by mathematical procedures, we will see him rely on fallacies and lock himself into real labyrinths. What we mean is that he will intend to introduce the fallacy of time dilation and the inappropriate use of the Lorentz factor intervention. Moving away from these false paths, we do not treat the expression $E=m c^{2}$ as a mathematical formula, but instead, we investigate it as an expression on how to group these three physical magnitudes: Energy-Mass-Speed of light, in order to produce a work. With this approach, we will see that $E=m c^{2}$ is the expression of the history of an atom and the manifestation of the energy that it is capable of supplying. For this, we have created the Theory of the Meeting (TM) that allows us to go to the origin of when this energy was generated. We leave for consideration, analysis, and criticism, for experts in astrophysics and atomic physics.
\end{abstract}

Keywords: Remote times, Energy-Mass-Speed, Theory of the Meeting (TM)

C2019 The Authors. Published by Fundamental Journals. This is an open access article under the CC BY-NC https://creativecommons.org/licenses/by-nc/4.0/

\section{https://doi.org/10.14331/ijfps.2019.330127}

\section{INTRODUCTION}

We begin with considering the idea of the movement of atoms, in infinite directions, within the matter that contains them. By applying our reasoning about the mass $m$ of an atom and, we anticipate in this brief summary, what we will later justify with physical expositions. The start point is from the fact that the expression $E=m c^{2}$ contains two physical magnitudes whose joint action in a certain process can generate a large amount of energy. From this fact, we will go back in time to try to find the path from where this source of energy began (Enrique Martinez Viladesau, 2018a).

In this return to the past and reinitiating the history of what happened, we hypothesized that a force $F$ of expansion intervened, perhaps due to an explosion, which in ancient times acted in all direction and on all atoms, between the atoms that we choose as a prototype in this paper. The force $F$ injected to the atom a potential to be able to develop a work. To identify this work $T$, we use the corresponding physical magnitudes that define as, 


$$
T=F e
$$

Here $F$ is the force that will have acted on the mass $m$ and $e$ is the space traveled by applying the aforementioned force. Therefore, the expression $E=m c^{2}$ shows that the above equation $\mathrm{Eq}(1)$ is the expression that manifests the origin and history of the atom. Here we present a theoretical approach that allows us to interpret our reasoning.

\section{THEORY OF THE MEETING (TM)}

In the development of this study, we present our theory, which we have called the Theory of the Meeting (TM) as follow. In fact, the expression $E=m c^{2}$ not be seen as a mathematical formula but as a physical expression that encompasses different concepts. For this reason, we will never use the wrong words of formula or equation, but we will use the word of expression. With this, we want to avoid the interpretation that when writing $E=m c^{2}$, we are interpreting it as a product of two variables, that is $E=m c^{2}$. To avoid such an interpretation, we need to consider that $E$ is reunited to the physical magnitudes, mass $m$ and speed of light $c$. Consequently, in symbolic form, we write,

$$
E=U m c^{2}
$$

Here we will define that, the expression of energy is equal to the reunion of mass and speed of light. To confirm our claim, we will inspect an atom containing this mass and identify it as the mass $m$ that we are testing.

\section{MANIFESTATIONS OF THE PHYSICAL MAGNITUDES}

To get to identify what the expression says that Eq (2) and continuing with the TM, we dealing with the information that a physical expression gives us, due to the relationships between the physical magnitudes that makes it up. We will accept that physical magnitudes, such as force, acceleration and ..., can detect their existence through the manifestations that can be observed in the experimental field. For example, the acceleration $a$ that acquires a mass $m$, will betray the existence of a force that causes it. Another aspect will be the assessment of its intensity, in which case we will apply the formula $F=m a$ but, according to what we said, we will now focus on considering the manifestations of the expressions of the physical quantities and avoid considering them as mathematical formulas that allow us to value them.

The approach of considering only the aforementioned manifestations, allows us to encompass and connect within the same physical phenomenon different physical magnitudes. This will allow us to interpret the meaning of expression $E=$ $U m c^{2}$. It allows us to go back from current data to the origins that produced them.

\section{LOOKING FOR THE SYMPTOMS OF EXPLOSION}

As previously mentioned, we started from the existence of an atom, which already existed in ancient times and we propose to relate the behavior of this atom in the current era, with what it expresses $E=m c^{2}$.

We will justify that the expression $E=U m c^{2}$ shows that the atom has incorporated the content of a force and this will lead us to admit there was a factor that caused the appearance of this force (Enrique Martinez Viladesau, 2018b).

We believe that this cause was an explosion that drove the atoms out of a common center. To justify that the said expression shows that the atom has incorporated the content of a force proceed as follows. We will use the TM and write as $E=\cup m c \cup c$. With this expression, we want to indicate that the meeting of two events is joined. On one hand, there is the reunion of the mass $m$ with the speed of light. On the other hand, another meeting is added to this event, expressed as the speed $c$ of the light. Here we investigate the meaning of the expression $E=m c^{2}$, which, as we shall see, leads us to reveal the existence of a force. In the previous issue, we have exposed the way in which the actions that perform the physical magnitudes are manifested. With the hypothesis that there was a big explosion, the first Physical magnitude that we will consider is force. The primogenital force acted on the atom at the origin of its existence. The physical magnitude that we are considering, by acting continuous on the particle, we know that it is manifested by the action of printing an acceleration $a$. Therefore, we can express this action as,

$$
F=U m a
$$

and, the acceleration $a$ is expressed as,

$$
a=U \frac{\partial v}{\partial t}
$$

That $v$ here is being the speed that the atom was acquiring due to the thrust of the force $F$ and $t$ the time employed. On the other hand, we know that by using mathematical terms we can consider the variation of that velocity $v$ due to the aforementioned force, by means of the formula.

$$
v=\int_{i}^{f} \frac{e}{t} d t
$$

where $v$ is the final speed that is to be reached after the time $t$ elapses between the initial and final points. However, we must bear in mind that the final point $f$ is located in the place where $v$ reaches the speed of light $c$, since this is the limit value of speed. Therefore, the acceleration between the starting point and the end point, that is, the increase in speed between zero and $c$, will be $\Delta v$ between 0 and $c$. So the referred force $F$ firstborn that acted on the particle must be expressed as,

$$
F=U m c
$$

Reasoning a way to the inverse of the one that we have used, we can say that the aforementioned expression tells us the story that there was a primogenital force due to that explosion, which ejected and pushed the atom we are analyzing.

\section{MODIFICATION THROUGH THE EXPRESSION OF THE ENERGY CONTAINING THE ATOM}

By investigating through the expression, $E=m c^{2}$ we show that the energy contained in the atom is manifested. Obtained the expression of the force $F$ that intervened, we must find out how the expression $E=U m c^{2}$ shows the capacity that the analyzed atom can perform a work $T$, that is, as the aforementioned expression states that the atom is in possession of supply a potential energy $E$. 
This is another conclusion to come from the aforementioned expression. We justify the possession of this energy because the atom at a moment of its journey acquired a kinetic energy. By not consuming it, it was accumulated as potential energy $E_{p}$, and this potential energy can be expressed as having trapped the ability to perform a work $T$. We remember the idea of movement, in all directions, of the atoms contained in the material. We will propose the equivalence between this energy $E_{p}$ and its ability to perform a job $T$. This is to say that,

$$
E_{p} \sim T
$$

Applying the topic that we discussed on part three we will assimilate the content in potential energy with the quality of performing a work. Applying physical concepts, to identify the work $(T)$, we will use the following reunion of physical magnitudes as,

$$
T=U F e
$$

That is, the work $T$ that the aforementioned expression states that the atom is capable of providing, has the atom saved and has it registered as the reunion of the first-born force $F$ of which we have spoken and $e$ the space that traveled the mass at the time when the events occurred. Recall that in the physical level, we measure the distances we move in light speed units $U v e$, so the referred particle, pushed by force $F$

\section{REFERENCES}

Viladesau, E. M. (2008). Theory of Relativity: LAP Lambert Academic Publishing Okt

Viladesau, E. M. (2018a). Theory of relativity-atomic watches and time dilation. International Journal of Fundamental Physical Sciences (IJFPS), 8(1), 1-4.

Viladesau, E. M. (2018b). Theory of relativity: Analysis of Lorentz transformation and Lorentz factor. International came to move at the speed of light $c$. In addition, with this form, we measure the routes $e$ (Enrique Martínez Viladesau, 2008; Enrique Martinez Viladesau, 2018c, 2019).

We value space $e$ in units of light speed $u v l$. If $v$ increases until reaching speed of light $c$, then is equivalent to having reached one unit $c$ so that $e=c$ and therefore, we have,

$$
T=U F C
$$

To be able to show that the expression $E U m c^{2}$ in its content contains the idea of a potential energy, we propose the following reasoning. As we have indicated $W U F C$ and how we have obtained, $F=U m c$ is obtained

$$
E \sim T=U(m c) U c=T U m c^{2}
$$

which is the expression we were investigating. Here we can observe that $E$ is seen as the reunion of a quantity of movement that takes the mass and the space $e$ that was displaced, when measuring it in units of light speed $u v l$, it is expressed with $\mathrm{c}$. In summary, the expression $E=m c^{2}$ placed in the test piece of analysis, and treated as the expression of a grouping of physical variables, informs us of what happened to the atom in ancient times and of what is able to perform. We leave the consideration, analysis and criticism, for the experts in astrophysics and atomic physics.

Journal of Fundamental Physical Sciences (IJFPS), 8(3), 87-91.

Viladesau, E. M. (2018c). Theory of special relativity: False premise that leads to obtain incorrect conclusions. International Journal of Fundamental Physical Sciences (IJFPS), 8(2), 83-86.

Viladesau, E. M. (2019). Theory of Relativity: The Fallacy of the Principle of Equivalence. International Journal of Fundamental Physical Sciences (IJFPS), 9(1), 6-9. 